\title{
FORAMINIFEROS PLANCTÔNICOS NO CRETÁCEO MÉDIO DA BACIA DE SANTOS, BRASIL
}

\author{
MARTA CLÁUDIA VIVIERS*
}

\begin{abstract}
Sediments of Late Albian-Coniacian age in the Santos Basin have yielded an abundant and relatively well preserved microfauna consisting of planktonic and bentonic foraminifers, ostracods, and radiolarians. Study of the planktonic foraminifers allows the definition of local biozones Rotalipora ticinensis, Dicarinella hagni and Marginotruncana undulata, the Whiteinella spp. interval and recognition of the classic Praeglobotruncana helvetica Zone. The aspects present in the sediments analysed are the same found elsewhere in basins developed in areas of the South and North Atlantic and Mesozoic Thetys oceans. This enables one to correlate these local zones with other, better-known ones of other countries. The more evident biostratigraphic relationships among data from planktonic foraminifers found in the studied interval are presented and discussed, as well as the relationships among the microfaunas known in the area of the early South Atlantic Ocean.
\end{abstract}

INTRODUÇÃo Recentemente a Petróleo Brasileiro S.A. realizou um programa de estudo geológico global da Bacia de Santos. $\mathrm{Na}$ análise estratigráfica desta bacia foi feita uma bioestratigrafia detalhada que envolveu foraminíferos planctônicos, palinomorfos e nanofósseis calcários. Tal investigação resultou no reconhecimento do arcabouço bioestratigráfico local, que pode, agora, ser comparado aos zoneamentos-padrão ou àqueles estabelecidos para outras bacias marginais do Atlântico Sul.

Este trabalho apresenta uma análise do conteúdo de foraminíferos planctônicos do intervalo Albiano Superior-Coniaciano, com enfoque especial para uma seqüência relativamente completa de associaçōes diagnósticas da passagem Cenomaniano-Turoniano a Turoniano Superior.

Foram investigados cerca de 37 poços e analisadas amostras de testemunhos e de calha, estas últimas tomadas a intervalos regulares de $30 \mathrm{~m}$.

\section{LOCALIZAÇÃO GEOGRÁFICA A Bacia da Santos} estende-se entre os paralelos $26^{\circ} 00^{\prime} \mathrm{S}$ e $23^{\circ} 00^{\prime} \mathrm{S}$, ao longo da costa dos Estados de Santa Catarina, Paraná, São Paulo e Rio de Janeiro. Os limites geológicos são o Alto de Florianópolis ao sul e o Arco de Cabo Frio ao norte. A área da bacia e as perfurações analisadas podem ser visualizadas na figura 1. Sua localização dentro do contexto do primitivo Oceano Atlântico Sul é mostrada na figura 2.

ESTRATIGRAFIA $O$ intervalo sedimentar estudado compreende o topo da Formação Guarujá e a totalidade da Formação Itajaí, como definiram Ojeda \& De Cesero (1973) e Ojeda \& Aranha (1980), respectivamente.

Formação Santa Catarina é outra denominação proposta para a última unidade estratigráfica (Williams \& Hubbard 1984).

A Formação Guarujá apresenta três seçōes bem definidas, que compreendem calcarenitos, calcilutitos e margas. Neste trabalho, foram analisadas, unicamente, as fácies de margas e de calcilutitos de plataforma profunda, correspon. dentes à parte superior da formação. Tal intervalo sedimentar apresenta uma microfauna planctônica relativamente desenvolvida e preservada.

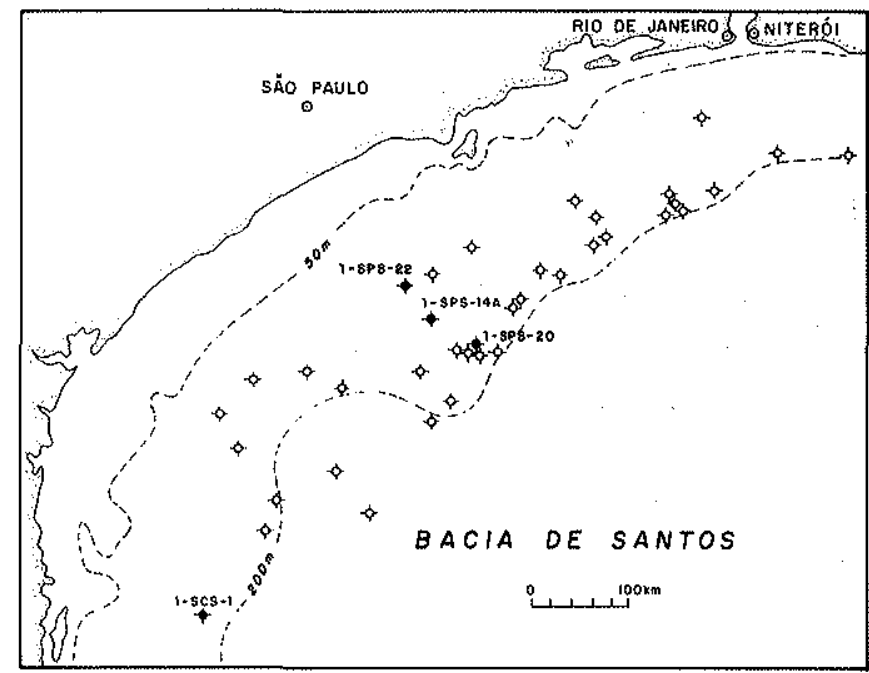

Figura 1-Mapa de situação dos poços citados no texto

A Formação Itajaí, constituída de sedimentos clásticos finos, foi o intervalo mais bem estudado em função da alta frequêencia de foraminíferos planctônicos encontrada. Esta unidade tem sua maior área de desenvolvimento na parte sul da bacia e na plataforma de Florianópolis; para o norte, as camadas diminuem de espessura gradativamente. Seu sistema deposicional é de plataforma-talude, que inclui fácies de prodelta e de clásticos finos de plataforma e de talude (Ojeda \& Aranha 1980).

BIOESTRATIGRAFIA A primeira informação detalhada acerca da bioestratigrafia da Bacia de Santos, a partir de análise micropaleontológica de perfurações, é de Koutsoukos (1982), que realizou um estudo geo-histórico e paleoecológico de quatro poços exploratórios desta bacia e da de Florianópolis, no intervalo Cretáceo-Terciário. Os trabalhos de Ojeda \& De Cesero (1973), Goncalves (1979), Ojeda \& Aranha (1980), Gibbons et al. (1983) e Williams \& Hubbard (1984), entre outros, são de índole estratigráfica, análise tectono-sedimentar baseada em dados 


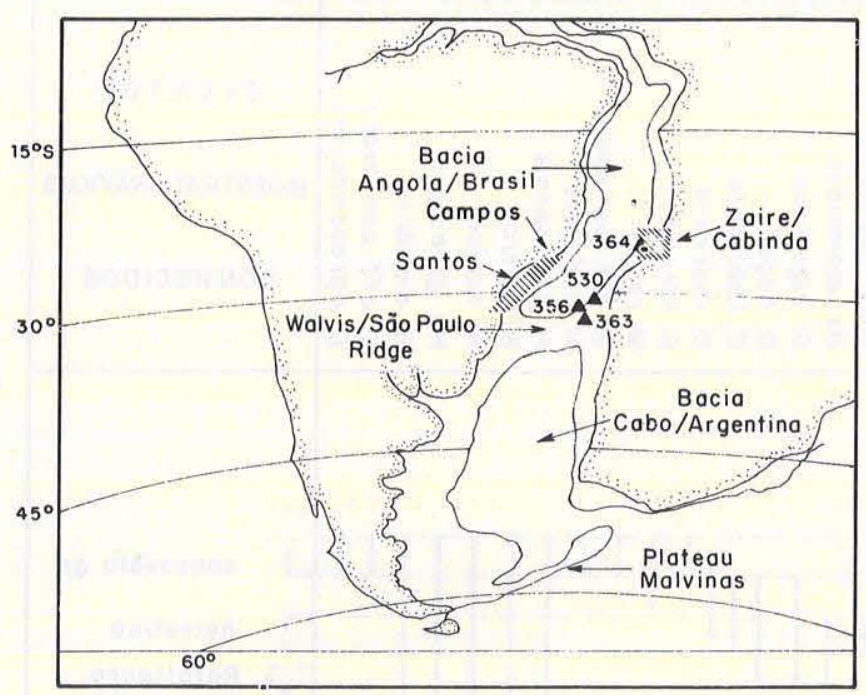

Figura 2 - Mapa mostrando as localizações relativas das perfurações do DSDP e das Bacias de Santos e Zaire/Cabinda. Reconstrução para \pm 100 e $\pm 10 \mathrm{Ma}$. Modificado de Sclater et al. (1977) e Dean et al. (1984)

sismoestratigráficos e geoquímica, que não fornecem maioies detalhes sobre os dados bicestratigráficos utilizados. No mencionado trabalho de Koutsoukos, a seqüência sedimentar pertencente à Formação Itajaí é datada como de idade cenomaniana-coniaciana no poço 1-SPS-14 e turoniana-santoniana nos poços 1-SCS-2 e 1-SCS-3, dados estes baseados na presença de faunas de foraminíferos planctônicos, nanoplâncton calcário e palinomorfos dessas idades. Sobre o contexto microfaunístico da Formação Guarujá, não existem dados publicados relacionados com foraminíferos planctônicos.

$\mathrm{O}$ arcabouço bioestratigráfico da Bacia de Santos, aqui definido e apresentado, foi elaborado a partir da integração dos dados micropaleontológicos obtidos com as informações disponíveis na literatura, correspondentes a zoneamentos diversos (Van Hinte 1976, Sigai 1977, Robaszynski \& Caron 1979, Wonders 1982, Robaszynski et al. 1982, Longoria 1984). Tal integração é possível já que na Bacia de Santos, no intervalo enfocado, ocorrem associações de espécies bem carácterísticas e de ampla difusão nos mares cretáceos. Conhecendo-se os estudos paleontológicos realizados, especialmente nas duas últimas décadas, em seções dos estratótipos cretáceos, que correlacionam esquemas zonais obtidos a partir de foraminíferos planctônicos, de nanofósseis calcários e o zoneamento estândar baseado em amonóides (Moullade 1966, Porthault 1969 e 1974, Seronie-Vivien 1972, Van Hinte 1976, Sigal 1977, Rat et al. 1979, Wonders 1980, Robaszynski et al. 1982), foi possível relacionar, com alto grau de ajuste, as unidades bioestratigráficas e cronoestratigráficas da Bacia de Santos com as reconhecidas no continente europeu. Por outro lado, com os resultados que vêm sendo divulgados pelo Deep Sea Drilling Project (DSDP) nos últimos anos, constata-se que a Bacia de Santos, para o intervalo estudado, continha uma fauna semelhante à de outras áreas do Oceano Atlântico Sul.

Žonas de foraminíferos planctônicos São definidas três biozonas: Zona Rotalipora ticinensis, para o Albiano Superior (Vraconiano); Zona Dicarinella hagni, para o Turoniano
Superior; e Zona Marginotruncana undulata, para o Coniaciano, além de ser reconhecida a Zona Praeglobotruncana helvetica, biozona clástica para o Turoniano Inferior-Médio e um intervalo informal, Intervalo Whiteinella spp. para o Cenomaniano (s.l.).

Por se tratar de estudo de amostras de calha, as zonas são do tipo "zona de intervalo", à exceção da Zona $P$. helvetica, definida pela amplitude local da espécie-guia, em amostras de testemunho.

No intervalo datado como Cenomaniano (s.l.) por palinologia (Intervalo $\gamma$ de Uesugui 1976), não foi possível caracterizar biozonas com utilização de foraminíferos plactônicos, já que a microfauna associada é de espécies de hedbergelídeos (Whiteinella, Hedbergella), muito monótona e geralmente mal preservada, cujas amplitudes ultrapassam os limites do andar.

Na figura 3 pode ser visualizada a distribuição estratigráfica das principais espécies de foraminíferos planctônicos encontradas na Bacia de Santos, e no quadro 1, as relações entre o zoneamento proposto e os utilizados para fins comparativos.

\section{ZONA Rotalipora ticinensis}

Definição: O topo da zona é caracterizado pelo nível local de desaparecimento de Rotalipora ticinensis, e a base, pelo nível local de desaparecimento do gênero Favusella.

Idade: Neoalbiano (Vraconiano) - (parte inferior).

Comentário: As camadas portadoras da fauna de Favusella spp. (primeira ocorrência na bacia de formas planctônicas bem características) e que definem a base da Zona $R$. ticinensis, situam-se num horizonte estratigraficamente mais alto que aquele no qual se extingue Cyclopsiella sp, palinomorfo, que nas bacias da margem leste determina o intervalo $\alpha$, indicativo do Eo-Mesoalbiano (Uesugui 1976, Viviers 1986).

Quanto ao topo da zona, a presença de $R$. ticinensis, encontrada junto a Ticinella raynaudi (s.l.) e Ticinella roberti, confirma uma idade Neo-Albiano (Vraconiano Inferior) para o mesmo, já que tanto a primeira espécie quanto o gênero Ticinella são formas que não alcançam o topo do Albiano. Rotalipora ticinensis é mencionada por Sigal (1977), Longoria (1984) e Martínez Gallegos (1984), em áreas do Mediterrâneo, México e Espanha, respectivamente, em associações com Planomalina buxtorfi, sem alcançar o nível de extinção desta última forma. O mesmo acontece com o gênero Ticinella (Sigal op. cit., Wonders 1980, Reyment 1982). Pelas relações bioestratigráficas mostradas, a Zona $R$. ticinensis estaria restrita à parte inferior do Albiano Superior (Vraconiano), sem alcançar a passagem Albiano-Cenomaniano.

Acima dos níveis em que está representada a Zona Rotalipora ticinensis existem camadas que contêm unicamente radiolários e uma microfauna bentônica relativamente diversificada. A extinção de Nannoconus truitti truitti, conhecida para o topo do Albiano Superior nas bacias da margem sudeste brasileira, ocorre neste intervalo. Isso poderia sugerir que o Albiano terminal também está representado, embora sem foraminíferos planctônicos característicos.

Equivalências:

- Zona Ticinella breggiensis e base da Zona Rotalipora appenninica/Planomalina buxtorfi (Sigal 1977).

- Zona Pseudothalmanniella ticinensis, Zona Planomalina praebuxtorfi e Zona Pseudothalmanniella ticinensis-Planomalina buxtorfi (Wonders 1980). 


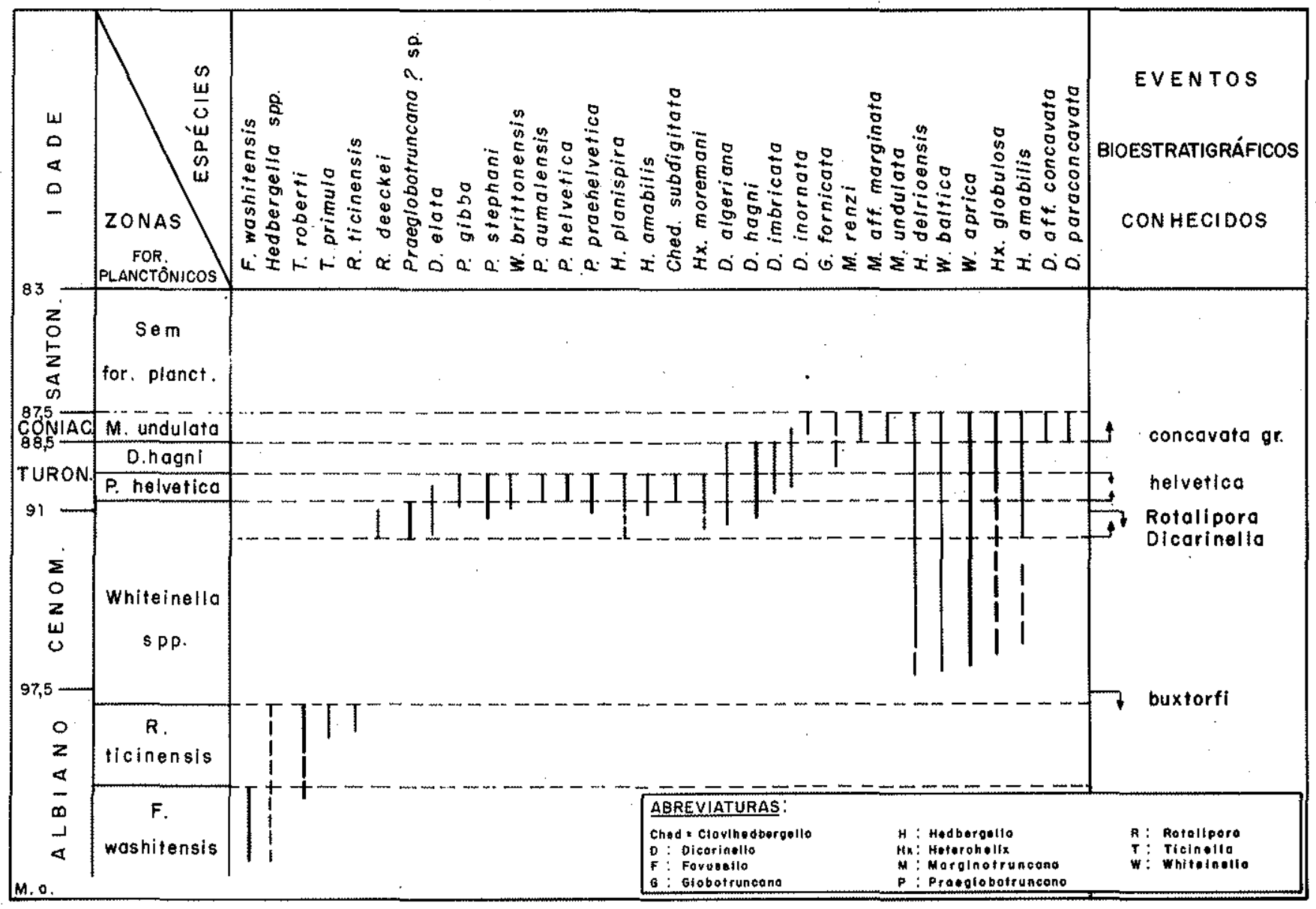

Figura 3 - Distribuição bioestratigráfica dos foraminiferos planctônicos na Bacia de Santos. Segundo Harland et al. (1982)

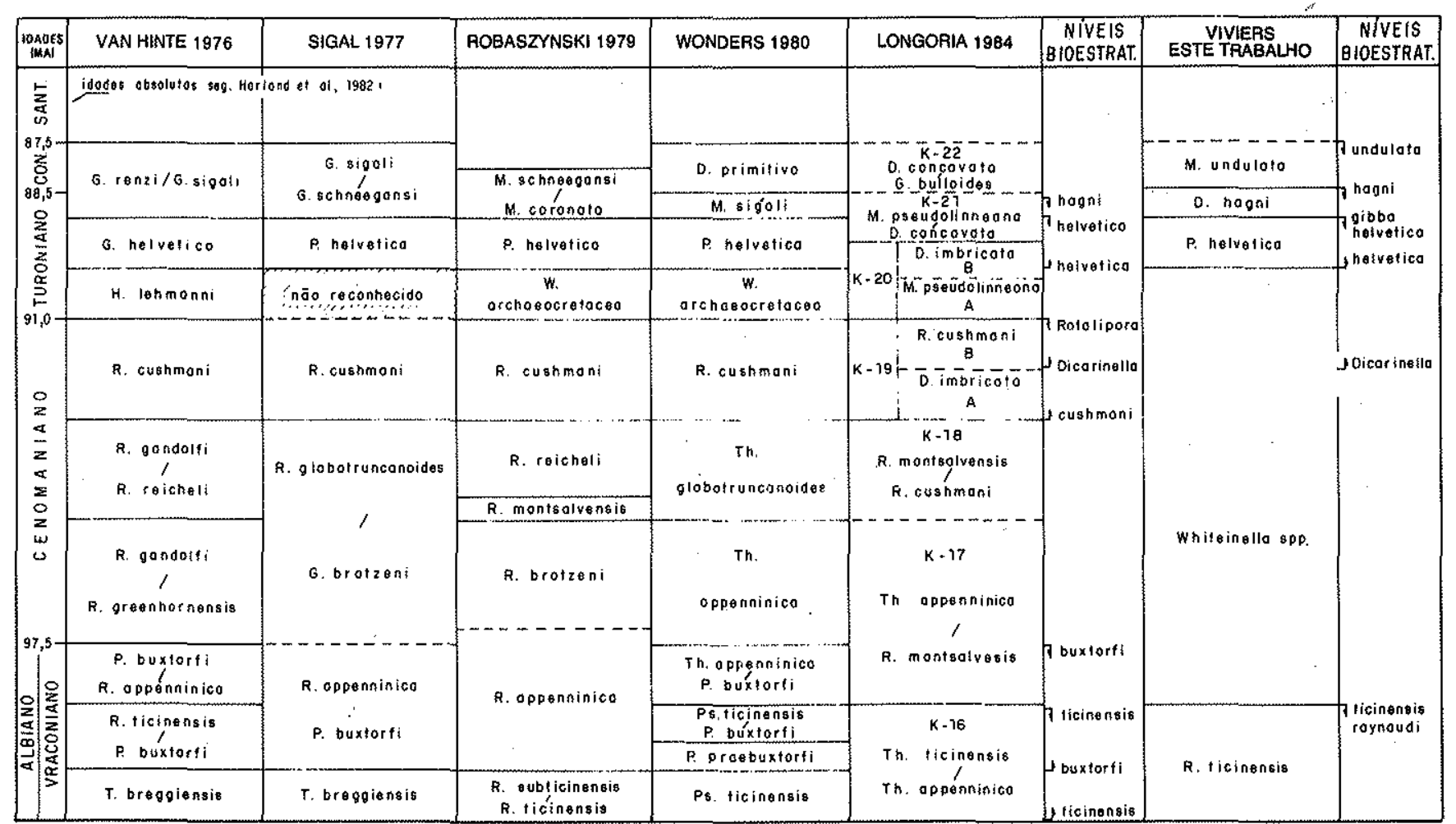

Quadro 1 - Comparaçäo do zoneamento proposto com os de outros autores. Cronoestratigrafia 
- Zona K 16 - Thalmanniella ticinensis-Thalmanniella appenninica e base da Zona K 17 - Thalmanniella appenninica/Rotalipora montsalvensis (Longoria 1984). Seção de referência: 1-SPS-20 (4.830-4.860 m).

\section{INTER VALO Whiteinella $\mathrm{sp} . \mathrm{sp}$.}

Definição: Intervalo compreendido entre o desaparecimento dos foraminíferos planctônicos albianos (Ticinella sp. sp., Rotalipora ticinensis) e a ocorrência de Praeglobotruncana helvética.

Idade: Cenomaniano (s.l.).

Comentário: Esta unidade está relacionada à ocorrência de elevada frequêencia de foraminíferos planctônicos hedbergeliformes grandes e pustulosos: Whiteinella aprica, $W$. baltica, $W$. archaeocretacea, Hedbergella delrioensis etc.

Dentro do intervalo, aparecem as primeiras formas planctônicas com carena dupla: Dicarinella hagni, D. imbricata, $D$. algeriana e $D$. elata. No processo evolutivo dos foraminíferos planctônicos, o aparecimento dessas formas bicarenadas é conhecido como anterior à extinção do gênero Rotalipora, ocorrido no topo do Cenomaniano (Porthault 1974, Longoria 1984).

$\mathrm{Na}$ Bacia de Santos, esta relação é difícil de estabelecer já que a presença das rotaliporas é pouco significativa. Rotalipora deekei foi reconhecida somente em uma perfuração. Por outro lado, o topo desta unidade bioestratigráfica é habitualmente relacionado à extinção do palinomorfo Classopollis major (Intervalo $\gamma$, de Uesugui 1976).

Em outras bacias sedimentares, o topo da associação de "grandes hedbergellas" está acima ou estreitamente relacionado com o nível de extinção de $C$. major (Bacia de Campos) e com o de Gnetaceapollenites similis (Bacia Potiguar). Esses palinomorfos marcam a passagem Cenomaniano-Turoniano nas bacias da margem leste e equatorial, respectivamente (Uesugui 1976, Azevedo et al. 1987, Regali \& Gonzaga 1985, Viviers \& Regali 1987).

Além disso, a ocorrência de Praeglobotruncana helvetica no topo da unidade é indicação suficiente para sugerir que este intervalo bioestratigráfico não atingiria uma idade mais nova que a do Turoniano basal.

Ante a ausência de espécies-guia diagnósticas no topo do intervalo, não é identificada uma localidade de referência.

Equivalências:

- Zona "Grandes Globigerines seules" (Lehmann 1963).

- Zona Praeglototruncana gigantea (Bolli 1966).

- Zona Hedbergella lehmanni (Van Hinte 1976, Premoli Silva \& Boersma 1977).

- Zona Whiteinella archaeocretacea (Robaszynski 1979, Wonders 1980).

- Zona Whiteinella brittonensis (Seiglie \& Baker 1982, Viviers \& Regali 1987).

\section{ZONA Praeglobotruncana helvética}

Definição: Esta zona é definida pela amplitude total da espécie-guia, tal como é reconhecido por praticamente todos os autores consultados.

Idade: Eo-Mesoturoniano.

Comentário: Dentro desta zona, desaparecem Praeglobotruncana stephani e $P$. gibba, fato mencionado também em outras áreas (Van Hinte 1976, Lamolda 1978). Pela primeira vez, nas bacias brasileiras, é possível caracterizar esta biozona com formas típicas do intervalo; uma menção anterior (Viviers 1982), na Bacia do Ceará, foi baseada em material muito escasso e mal preservado.
Equivalências: Esta unidade é equivalente à biozona homônima de quase todos os autores consultados.

Localidade de referência: Poço 1-SCS-1 (3.309 a 3.326 m).

\section{ZONA Dicarinella hagni}

Definição: O topo da zona é definido pelo nível de desaparecimento local de Dicarinella hagni e a base, pelo nível de extinção de $P$. helvética.

Idade: Neoturoniano.

Comentário: Nesta unidade bioestratigráfica coexistem formas carenadas típicas do Turoniano: Dicarinella hagni, D. imbricata, $D$. algeriana, além de Marginotruncana renzi, e hedbergelídeos vários (Hedbergella delrioensis, Whiteinella aprica, $W$. baltica etc.). A participação das últimas formas no total da associação é bem menos importante que nas outras biozonas do Cenomaniano-Turoniano Inferior.

Acompanhando a fauna planctônica, é comum encontrar-se o ostracode Brachycythere sapucariensis.

A idade Neoturoniano da zona é baseada nas relações estratigráficas entre o nível de extinção de $P$. helvetica (durante o Mesoturoniano) e as extinções de $D$. hagni, $D$. imbricata e $D$. algeriana, espécies que dificilmente alcançam o Coniaciano (Robaszynski \& Caron 1979).

Equivalências: Esta zona é equivalente à parte inferior de: - Zona Globotruncana renzi/Globotruncana sigali (Van Hinte 1976).

- Zona Globotruncana sigali/Globotruncana schneegansi (Sigal 1977).

- Zona Marginotruncana sigali (Wonders 1980).

Seção de referência: 1-SPS-22 (2.208 a 2.415 m).

\section{ZONA Marginotruncana undulata}

Definição: Topo da zona definida pelo nível de extinção de Marginotruncana undulata e a base, pelo nível local de extinção de Dicarinella hagni.

Idade: Coniaciano (s.l.).

Comentário: Não foi possível caracterizar esta unidade com muitos elementos já que a seqüência sedimentar em que está representada se encontra desenvolvida em ambientes relativamente rasos.

O topo da zona coincide praticamente com o nível de desaparecimento dos foraminíferos planctônicos na área de estudo. Tal evento "catastrófico" esteve associado a uma sensível mudança paleoambiental, relacionada, provavelmente, com um rebaixamento relativo do nível do mar.

A ausência quase total de formas planctônicas de Dicarinella concavata (espécie típica do Coniaciano-Santoniano, de águas profundas) pode estar relacionada tanto a razões paleobatimétricas (águas relativamente rasas) quanto ao fato de o intervalo considerado estar restrito à transição Turoniano-Coniaciano ou ao Coniaciano basal.

Em virtude das escassas informações fornecidas pelos foraminíferos planctônicos, considera-se esta zona de idade coniaciana (s.l.). Portanto não são estabelecidas relações com outros zoneamentos e não é definida uma seção de referência.

OBSERVAÇÕES BIOESTRATIGRAFICAS E CRONOESTRATIGRÁFICAS A definição das novas zonas nos depósitos do Mesocretáceo da Bacia de Santos evidencia uma sucessão de eventos bioestratigráficos importantes, por se tratar de níveis de aparecimento e desaparecimento evolutivos de gêneros e espécies de foraminíferos plactônicos bem conhecidos. Alguns deles constituem níveis de 
fácil correlação regional na área da bacia e de outras bacias da margem continental brasileira (Viviers \& Beurlen 1987).

Esses eventos são comparados com acontecimentos similares ocorridos em outras áreas, onde, por terem sido relacionados com zoneamentos de amonóides, possuem uma posição cronoestratigráfica mundialmente aceita. Alguns deles também foram detectados em outras bacias brasileiras (Viviers 1985, Viviers \& Regali 1987, Azevedo et al. 1987). Em muitos casos, servem como critérios para reconhecer limites de andares ou parte deles (Viviers \&Beurlen 1987). Os que ocorrem na Bacia de Santos são os seguintes:

a) Desaparecimento do gênero Favusella - base do Albiano Superior.

b) Desaparecimento praticamente simultâneo de Rotalipora ticinensis, Ticinella roberti, $T$. raynaudi - parte inferior do Vraconiano (Albiano Superior).

c) Aparecimento das primeiras formas planctônicas bicarenadas: Dicarinella hagni, D. imbricata - Neocenomaniano. d) Desaparecimento de Praeglobotruncana helvetica, $P$. stephani e $P$. gibba - Mesoturoniano.

e) Desaparecimento de'Dicarinella hagni - Neoturoniano.

A ocorrência de Rotalipora ticinensis em níveis com Ticinella roberti e $T$. raynaudi serve para localizar a porção inferior do Vraconiano. Tal fato é mencionado por Wonders (1980), que registra o desaparecimento de $R$. ticinensis, uma das primeiras rotaliporas conhecidas, no Vraconiano (Zona Rotalipora ticinensis-Planomalina buxtorfi). Nos mesmos níveis estratigráficos também desaparece o gênero Ticinella (Wonders 1980, Longoria 1984).

Nas análises realizadas, foram detectadas o aparecimento das primeiras formas bicarenadas (Dicarinella hagni, $D$. algeriana) e a ocorrência de Praeglobotruncana helvetica. O primeiro evento, segundo Porthault (1974), ocorre na parte basal do Cenomaniano Superior; Longoria (1984) registra-o em níveis prévios à extinção do gênero Rotalipora (topo do Cenomaniano Superior). Ante a ausência de outras indicações, este evento é associado ao Neocenomaniano.

Outros eventos - como a ocorrência de $P$. helvetica, geralmente associada a $P$. praehelvetica e $P$. gibba, e as extinções praticamente contemporâneas desses três taxa e de Dicarinella hagni - são as melhores indicações para reconhecer o Turoniano Inferior a Médio, o topo do Turoniano Médio e o Turoniano Superior, respectivamente. Estes são data mundialmente aceitos, já que se baseiam em espécies encontradas associadas a amonóides em camadas do estratótipo turoniano (Robaszynski et al. 1982).

O ATLÂNTICO SUL NO MESOCRETÁCEO O estudo dos foraminíferos planctônicos do Mesocretáceo das bacias marginais do Atlântico Sul e de áreas profundas perfuradas pelo DSDP oferece um amplo conjunto de dados que permite o reconhecimento de eventos bioestratigráficos globais significativos na história deste oceano.

As associações que ocorrem nas áreas mostradas na figura 2 apresentam praticamente as mesmas espécies. As descontinuidades do registro sedimentar se dão, também, em intervalos equivalentes.

$\mathrm{Na}$ figura 4, são comparadas as seqüências de biozonas encontradas nas diferentes localidades estudadas no Atlântico Sul: margem oeste, bacias de Santos (este trabalho) e Campos (Koutsoukos 1984, Azevedo et al. 1987); área central - Site 356 (Premoli Silva \& Boersma 1977); e margem leste - Site 530A (McNulty 1984), Sites 363 e 364 (Caron 1978) e bacias do Zaire e de Cabinda (Seiglie \&
Baker 1982). Pode ser visualizada, também, a magnitude dos hiatos identificados em todas as áreas consideradas.

Durante o Neo-Álbiano a microfauna planctônica apresenta caracteres bem semelhantes, destacando-se 0 predomínio de formas hedbergeliformes (Hedbergella, Ticinella, Globigerinelloides) e a escassa participação das formas carenadas. Entre estas, uma das espécies mais mencionadas é Rotalipora ticinensis, que está entre as formas mais primitivas de rotaliporas. Em algumas das áreas marginais das bacias de Santos, Zaire e Cabinda, a falta de componentes planctônicos nas associações é interpretada como produto de condições paleobatimétricas muito rasas, imperantes durante aquele tempo, nos referidos sítios sedimentares. Como única diferença, cabe mencionar a ocorrência de Favusella washitensis em intervalos restritos ao Albiano na margem oeste, que, no Zaire e em Cabinda, alcança o Cenomaniano Inferior.

O Cenomaniano está representado nas áreas cie ambas as plataformas costeiras com associações bem características, sempre de hedbergelídeos (Hedbergella, Whiteinella). $\mathrm{O}$ registro de planctônicos carenados neste intervalo também é reduzido em ambas as margens. Na Bacia de Santos, uma das bacias brasileiras na qual se registrou até o momento a presença do gênero Rotalipora no Cenomaniano, Rotalipora deeckei ocorre em estratos do Cenomaniano Superior, assim como também é detectada nela a presença dos primeiros foraminíferos planctônicos dicarenados (Dicarinella hagni e $D$. algeriana).

Já na margem leste, as rotaliporas foram registradas em sedimentos do Cenomaniano Inferior $(R$. appenninica, $R$. brotzeni) e do Cenomaniano Superior ( $R$. greenhornensis) (Seiglie \& Baker 1982).

Na áre: central do primitivo Oceano Atlântico Sul, até o momento não foram identificacias microfaunas tipicamente cenomanianas. Por via de regra, para esse tempo, a ausência dessas faunas está relacionada com um hiato expressivo, que coloca sedinentos do Albiano Superior em contato com os de idade turoniana ou coniaciana (Fig. 4).

A origem deste hiato pode estar ligada a episódios erosivos provocados por correntes de fundo, em resposta a alterações no regime circulatório das águas (Butt 1982, Dias-Brito 1987). Nas bacias marginais, o hiato não é tão evidente ou é menos expressivo, faltando, às vezes, seções do Cenomaniano; entretanto, fortes alterações do conteúdo microfaunístico são detectadas na passagem Albiano-Cenomaniano, em resposta a sensíveis mudanças que ocorreram neste tempo no paleoambiente deposicional (Dias-Brito op. cit., Koutsoukos 1984, Azevedo et al. 1985, Viviers et al. 1985). Isso resultou na ocorrência de camadas praticamente estéreis em fauna bentônica acima de um intervalo correspondente ao Neo-Albiano, tempo em que dominava uma microfauna de planctônicos (foraminíferos, radiolários) e de foraminíferos bentônicos, relativamente rica e diversificada. Tai mudança pode ainda estar relacionada com um ciclo de expansão dos mares cretáceos, que, ao invadirem áreas costeiras, remobilizaram elevadas quantidades de material clástico e modificaram acentuadamente as condições de fundo das plataformas.

$\mathrm{O}$ intervalo correspondente ao Turoniano-Santoniano é identificado micropaleontologicamente pela ocorrência de várias espécies diagnósticas: Praeglobotruncana heivetica e $P$. inornata, Dicarinella hagni, $D$. gr. concavata e D. imbricata. Tais espécies estão presentes praticamente em quase 


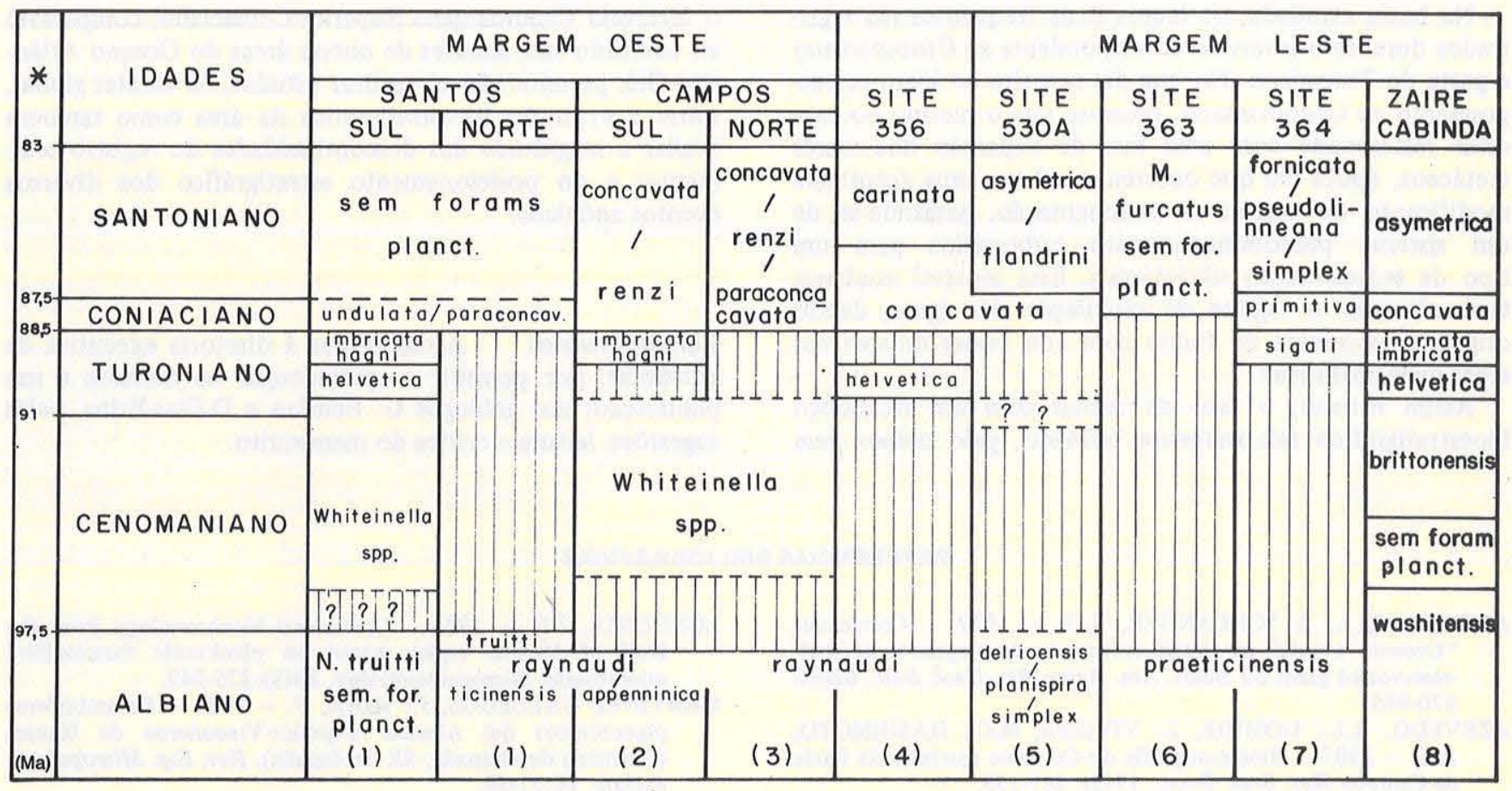

Dados obtidos de 1) Viviers et al. (1985); 2) Azevedo et al. (1987); 3) Koutsoukos (1984); 4) Premoli Silva \& Boersma (1977); 5) McNulty (1984); 6 e 7) Caron (1978); 8) Seiglie \&Baker (1985). Abreviaturas: N - Nannoconus; M - Marthasterites; e * Ma segundo Harland et al. (1982).

\section{Figura 4 - Albiano - Santoniano no Atlântico Sul:zonas de foraminiferos planctônicos. Hiatos}

todas as áreas analisadas. No entanto, na Bacia de Santos não foi possível caracterizar o Santoniano por meio de foraminíferos planctônicos, pois seqüências desta idade estão desenvolvidas em ambiente marginal, desprovidas de fauna tipicamente marinha (Viviers et al. 1985). Dentro deste intervalo, os hiatos mais freqüentes foram registrados no Neoturoniano.

Ainda quanto a importantes eventos na geo-história do Mesocretáceo do Atlântico Sul, destaca-se a ocorrência de episódios anóxicos globais. Na Bacia de Santos, são registrados no intervalo Cenomaniano-Turoniano, onde sedimentos com teores de carbono orgânico entre $1 \%$ e $2,5 \%$ (Gibbons et al. 1983), com populações muito ricas de foraminíferos planctônicos hedbergeliformes, estão desprovidos de fauna bentônica, interpretando-se que foram depositados sob um padrão de circulação de águas de fundo com caracteres de restrição.

Episódios de tais características também foram detectados em outras áreas do Atlântico Sul (Thiede \& Van Andel 1977, Dean et al. 1984), onde foram registrados valores de carbono orgânico próximos de $10 \%$. Este evento anóxico detectado na Bacia de Santos poderia, em parte, ser relacionado com o "Evento Anóxico 2", descrito por Arthur \& Schlanger (1979), para a passagem Cenomaniano-Turoniano, e que, dadas as características geológicas da área, pode ter continuado talvez até o Coniaciano.

CONCLUSÕES O estudo detalhado do conteúdo de foraminíferos planctônicos do intervalo Albiano Superior-Coniaciano da Bacia de Santos permitiu a elaboração de um arcabouço bioestratigráfico constituído de quatro zonas de um intervalo informal: Zona Rotalipora ticinen- sis - Albiano Superior (Vraconiano); Zona Praeglobotruncana helvetica - Turoniano Inferior-Médio; Zona Dicarinella hagni - Turoniano Superior; e Zona Marginotruncana undulata - Coniaciano - e intervalo Whiteinella spp. Cenomaniano. Parte deste zoneamento, por estar baseado na ocorrência de grupos de espécies bem conhecidas e de ampla difusão nos mares cretáceos, tem forte ajuste com os elaborados em outras áreas dos continentes europeu e americano.

Níveis de aparecimento evolutivo e extinção de algumas espécies-guia de foraminíferos planctônicos são relacionados estratigraficamente, representando uma seqüência de eventos bioestratigráficos, utilizáveis em correlações inter-regionais. São eles: desaparição do gênero Favusella - base do Albiano Superior (s.s.); desaparecimento simultâneo de Rotalipora ticinensis, Ticinella raynaudi e Ticinella roberti - Vraconiano; aparecimento das primeiras formas planctônicas com carena dupla e freqüência máxima de hedbergelídeos grandes e pustulosos - passagem Cenomaniano Superior-Turoniano; desaparecimento de Praeglobotruncana helvetica - Turoniano Médio; e desaparecimento de Dicarinella hagni - Turoniano Superior.

As associações de foraminíferos planctônicos do Mesocretáceo, identificadas na área da Bacia de Santos, são praticamente as mesmas que as reconhecidas em outras áreas do Oceano Atlântico Sul para esse mesmo intervalo. É possível, portanto, estabelecer relações bioestratigráficas bastante estreitas entre os depósitos sedimentares de praticamente toda a área central do primitivo oceano e as respectivas margens. É de se detacar que seqüências bioestratigráficas relativamente completas deste intervalo, no Atlântico Sul, são registradas nas bacias marginais de Santos, Zaire e Cabinda. 
$\mathrm{Na}$ bacia estudada, os hiatos mais freqüientes são registrados durante o intervalo correspondente ao Cenomaniano e parte do Turoniano. No que diz respeito ao hiato correspondente ao Cenomaniano, pensa-se que o mesmo poderia estar relacionado com uma fase de expansão dos mares cretáceos, época em que ocorreu, também, uma acentuada modificação do regime de sedimentação, passando-se de um sistema predominantemente carbonático para um tipo de sedimentação siliciclástica. Esta sensível mudança teria alterado o regime de circulação das águas, dando origem a correntes de fundo com alto poder erosivo nas áreas mais profundas.

Assim mesmo, o fato dé contar com um arcabouço bioestratigráfico relativamente refinado, pelo menos para o intervalo Cenomaniano Superior-Coniaciano, comparável ao utilizado nas análises de outras áreas do Oceano Atlântico Sul, permite não só realizar estudos, de caráter global, sobre a evolução bioestratigráfica da área como também avaliar a magnitude das descontinuidades do registro sedimentar e do posicionamento estratigráfico dos diversos eventos anóxicos.

Agradecimentos Agradecemos à diretoria executiva da Petrobrás, por permitir a apresentação do trabalho e sua publicação; aos geólogos G. Beưrlen e D.Dias-Brito, pelas sugestőes, leitura e crítica do manuscrito.

\section{REFERENCIAS BIBLIOGRAFICAS}

ARTHUR, M.A. \& SCHLANGER, S.O. - 1979 - Cretaceous "Oceanic events" as causal factors in development of reef-reservoired giant oil fields. Am. Assoc. Pet. Geol. Bull., 63(6): 870-885.

AZEVEDO, R.L.; GOMIDE, J.; VIVIERS, M.C.; HASHIMOTO, A.T. - 1987 - Bioestratigrafia do Cretáceo marinho da Bacia de Campos. Rev. Bras. Geoc., 17(2): 147-153.

BOLLI, H.M. - 1966 - Zonation of the Cretaceous to Pliocene marine sediments based on planktonic foraminifera. Bol. Inf. Assoc. Venez. Geol. Min. Petr., 9:3.32.

BUTT, A. - 1982 - Micropaleontological bathymetry of the Cretaceous of Western Morocco. Paleogeogr., Paleoclimat., Paleoecol., 37(2/4):235-276.

CARON, M. - 1978 - Cretaceous planktonic foraminifera from DSDP Leg 40, Southeastern Atlantic Ocean. In: BOLLI, H.M.; RYAN, W.B.E. et al. Init. Repts. DSDP, 40, Washington, (U.S. Govt. Printing Office), p. $651-678$.

DEAN, W.E.; HAY, W.W.; SIBUET, J.C. - 1984 - Geological evoluiton, sedimentation and paleoenvironments of the Angola Basin and adjacent Walwis Ridge, synthesis of results of DSDP. Leg 75. In: : HAY, W.W. SIBUET, J.C., et al. Intt. Reps. DSDP, 75, Washington, (U.S. Govt. Printing Office), p. 509-544.

DEAN, W.E.; ARTHUR, M.A.; STOW, D.A. - 1984 - Origin and geochemistry of Cretaceous deep-sea blackshales and multicolored claystones, with emphasis on Deep Sea Drilling Project-Site 530. Southern Angola Basin. In: HAY, W.W.; SIBUET, J.C; et al. Init. Repts. DSDP, 75, Washington, (U.S. Govt. Printing Office), p. 819-844.

DIAS-BRITO, D. - 1987 - A Bacia de Campos no Mesocretáceo: uma contribuição à paleoceanografia do Atlântico Sul primitivo. Rev, Bras. Geoc., 17(2): 162-167.

GIBBONS, M.J.; WILLIAMS, A.R.; PIGGOT, N.; WILLIAMS, G.M. - 1983 - Petroleum geochemistry of the Southern. Santos Basin, offshore Brazil. J. Geol. Soc. London, 140:423-430.

GONÇALVES, A. - 1981 - Geologia da Bacia de Santos. Rio de Janeiro, Petrobrás (Rel. int.).

HARLAND, W.; COX, A.V.; LLEWELLYN, P.G.; PICKTON, A.G.; SMITH, A.G.; WALTERS, R. - 1982 - A geological time scale. Cambridge, Cambridge University Press. (Cambridge Earth Science series).

KOUTSOUKOS, E.A.M. - 1982 - Geo-história e paleoecologia das bacias marginais de Florianópolis e Santos. In: CONGR. BRAS. GEOL., 32, Salvador, 1982. Anais... Salvador, SBG. v. 5, p. 2369-2382.

KOUTSOUKOS, E.A.M. - 1984 - Evolução paleoecológica do Albiano ao Maastrichtiano na área noroeste da Bacia de Campos, com base em foraminiferos. In: CONGR. BRAS. GEOL., 33, Rio de Janeiro, 1984. Anais... Rio de Janeiro, SBG. v. 2 , p. $685-698$.

LAMOLDA, M, - 1978 - Biozonas de foraminifèros planctónicos en el Turoniense Vasco-Cantabrico (Norte de España). Rev. Esp. Paleont., $9(2): 221-234$.

LEHMANN, R. - 1963 - Etude des Globotruncanidae du Crétacé Supérieur de la province de Tarfaya (Maroc Occidental). Notes Mem. Serv. Geol. Maroc., 21(156):133-181.
LONGORIA, J.F. - 1984 - Cretaceous biochronology from the Gulf of Mexico region based on planktonic foraminifera microfossils. Micropaleontology, 30(3):225-242.

MARTINEZ GALLEGOS, J,; MIRA, F. - 1984 - Foraminíferos planctónicos del Albense Superior-Vraconense de Huesca (Provincia de Granada, SE de Espaffa). Rev. Esp. Micropaleontologia, 16:51-58.

McNULTY, C.L. - 1984 - Cretaceous foraminifers of Hole 530A, Leg 75, Deep Sea Drilling Project. In: HAY, W.W.; SIBUET, J.C. et al. Init. Repts. DSDP, 75, Washington (U.S. Govt. Printing Office), p. 547-564.

MOULLADE, M. - 1966 - Etude stratigraphique et micropaleontologique du Crétacé Inférieur de la "Fosse Vocontlente". Lyon, Department des Sciences de la Terre de la Faculté des Sciences. 369 p. (Documents des laboratoires de géologie de la Faculté de Sciences de la Terre, 15).

OJEDA, H.A.O. \& ARANHA, L. - 1980 - Bacia de Santos. Integração geológica e regional. Rio de Janeiro, Petrobrás (Rel. int.).

OJEDA, H.A.O. \& DE CESERO, P. - 1973 - Bacias de Santos e Pelotas. Geologia e perspectivas petrolfferas. In: Petro* brás.Dexpro.Divex. Bacias da costa leste do Brasil; geologia e perspectivas petroliferas. Rio de Janeiro, p. 1-34 (Petrobrás).

PORTHAULT, B. - 1969 - Foraminifères planctoniques et bioestratigraphie du Cénomanien dans le Sud-Est de la France. In: CONFERENCIA INTERNACIONAL SOBRE MICROFOSSEIS PLANCTONICOS. 1, Genebra, 1967. Proceedings... Leiden, E.J. Buli. p. 526-546. 2 plates.

PORTHAULT, B. - 1974 - Le Crétacé Supérieur de la "Fosse Vocontiene" et des regions limitrophes (France Sud-Est), micropaléontologie, stratigraphie, paléogeographie. Lyon, Université Claude Bernard. 342 p. These Doot. (apud Longorfa, 1984).

PREMOLI SILVA, I. \& BOERSMA, A. - 1977 - Cretaceous planktonic foraminifera DSDP. Leg 39 (South Atlantic). In: SUPKO, P.R.; PERCH-NIELSEN, K. et al. Init. Repts. DSDP, 39, Washington, (U.S. Govt. Printing Office), p. 615-641.

RAT, P.; MAGNIEZ-JANNIN, F.; CHATEUNEUF, J.J.; DAMOTTE, R.; DESTOMBES, P.; FALCONNIER, D.; FEULLIERE, P.; MANIVIT, H.M.; MONGIN, D.; ODIN, G. - 1979 - Les stratotypes francais. L'Aubien de l'Aube. Paris, CNRS, $446 \mathrm{p}$.

REGALI, M.P.S. \& GONZAGA, S. - 1985 - Palinoestratigrafia da Bacia Potiguar. In: Brasil. MME-DNPM. Col. Trab. Paleont., Brasilia, Geologia 27 - Paleontologia e Estratigrafia, 2: 443.460 .

REGALI, M.P.S.; UESUGUI, N.; LIMA, E.C. - 1985 - Palinoestratigrafia da Bacia de Barreirinhas. In: Brasil. MME-DNPM, Col. Trab. Paleont. Brasília. Geologia 27. Paleontologia e Estratigrafia, 2:461-480.

REYMENT, R. - 1983 - The events of the Mid-Cretaceous. In: Stratigraphy quo vadis? Tulsa. AAPG. Studies in Geology, no 16; IUGS Special Publication no 14, p. 44-48.

ROBASZYNSKI, F. \& CARON, M., coord. - 1979 - Atlas de foraminifêres planktoniques du Crétacé Moyen (Mer Borel et Tethys). Cahters de Micropaleontologie, CNRS, 1979/1, 185 p.; $1979 / 2,181 \mathrm{p}$. 
ROBASZYNSKI, F.; ALCAYDE, G.; AMEDRO, F.; BADILLEY, G.; DAMOTIE, R.; FOUCHER, A.C.; JARDINEI, S.; LEGOUX, O.; MANIVIT, H.; MONCIARDINI, C.H.; SORNAY, J. - 1982 - Le Turonien de la région-type: Saumurois et Touraine: Stratigraphie, biozonation, sedimentologie. Bull. Centr. Rech. Explor. Prod. Elf-Aquitaine, 6(11): 119-225.

SCLATER, J.; HOLLINGER, S.; TAPSCOTT, C. - 1977 - The paleobatymetry of the Atlantic Ocean from the Jurassic to Recent. J. Geol., 35(5):509-552.

SEIGLE, G.A. \& BAKER, M.B. - 1982 - Foraminiferal zonation of the Cretaceous of Zaire and Cabinda, west Africa and its geological significance. Tulsa. AAPG. p. 651-658 (AAPG Memoir 34).

SERONIE-VIVIEN, M. - 1972 - Contribution a l'étude du Senonien en Aquitaine septentrional, des stratotypes: Coniacien, Santonien, Campanien. Paris, CNRS. 195 p.

SIGAL, J. - 1977 - Essai de zonation du Crétacé Méditerranéen a l'aide des foraminifères planctoniques. Geol. Méditerr., 2: 99-108.

THIEDE, J.C. \& VAN ANDEL, T. - 1977 - The paleoenvironment of anaerobic sediments in the Late Mesozoic South Atlantic Ocean. Earth Planet. Sci. Lett., 33:103-109.

UESUGUI, N. - 1976 - Intervalos bioestratigráficos da Bacia de Campos do Albiano ao Santoniano, Rio de Janeiro, Petrobrás, (Rel. int.).

VAN HINTE, J.E. - 1976 - Cretaceous time scale. Am. Assoc. Pet. Geol. Bull., 60(4):498-516.
VIVIERS, M.C. - 1982 - Biocronoestratigrafia da Bacia do Ceará. In: CONGR. BRAS. GEOL., 32, Salvador, 1982, Anais... Salvador, SBG. v. 5, p. 2433-2449.

VIVIERS, M.C. - 1985 - Características bioestratigráficas dos sedimentos Albo/Cenomanianos da Bacia do Ceará. Relações com outras bacias brasileiras e africanas. In: Brasil. MME-DNPM, Col. Trab. Paleont. Brasília. Brasília - Geologia 27; Paleontologia e Estratigrafia 2:529-537.

VIVIERS, M.C. \& BEURLEN, G. - 1987 - Sobre alguns níveis de correlação bioestratigráfica regional (foraminíferos planctônicos) no Cretáceo brasileiro. Rev. Bras. Geoc. 17(2):106-110.

VIVIERS, M.C. \&REGALI, M.P.S. - 1987 - Estudo paleoambiental preliminar no Cretáceo da Bacia Potiguar. Rev. Bras. Geoc. 17(2):123-130.

VIVIERS, M.C.; UESUGUI, N.; RICHTER, A.; PRAÇA, U.M.; HASHIMOTO, A.T.; ANTUNES, R.; HERTER, G. - 1986 Biostratigraphy and paleoecological evolution of the Middle and Upper Cretaceous of the Santos Basin, Brasil. An. Acad. bras. Ciênc., 58:172-173.

WILLIAMS, B.G. \& HUBBARD, R.J. - 1984 - Seismic stratigraphic framework and depositional sequences in the Santos Basin, Brazil. Mar. Pet. Geol., 1:90-104.

WONDERS, A.A.H. - 1980 - Middle and Late Cretaceous planktonic foraminifers of the Western Mediterranean area. Utrecht Micropaleontology Bulletin, 24:117 p.

MANUSCRITO 386

Recebido em 01 de setembro de 1986 Revisão aceita em 30 de março de 1987

"Salvo algumas passíveis exceções, o arcabouço tecnológico da 'terceira onda' tenderá, fatalmente, a diminuir a importância das matérias-primas, dentro da nova estrutura industrial, marcada pelo baixo consumo energético e pelo alto apelo à tecnologia da vanguarda". 